Rees, R. J. W. \& Hart, P. D'A. (1952). J. gen. Microbiol. 7, 372-381.

\title{
The Inhibition of Growth of Avirulent Strains of Mycobacterium tuberculosis by a Surface-active Polyoxyethylene Ether
}

\author{
By R. J. W. REES AND P. D'ARCY HART \\ The National Institute for Medical Research, Mill Hill, London, N.W. 7
}

SUMMARY : Examination of the action of a series of surface-active polyoxyethylene ethers on the growth of the avirulent strain of Mycobacterium tuberculosis, H37 Ra, showed that one member, the ether of $2,2^{\prime}$-dihydroxy-5, 5'-di-tert-octyldiphenylmethane (D4), inhibited this strain but not the virulent variant H37 Rv. This type of differential inhibition has been observed with a commercial surface-active mixture, 'Triton A 20' (Dubos \& Middlebrook, 1948). Lengthening of the polyoxyethylene chains of D4 led to loss of its inhibitory property. Both D4 and 'Triton A20' could induce resistance in $\mathrm{H37} \mathrm{Ra}$, and cross-resistance to $\mathrm{D} 4$ of a strain resistant to 'Triton A20' was observed; this cross-resistance suggested that a compound closely related to D4 was the active constituent of 'Triton A20'. The resistant strains grew in a microscopically amorphous pattern, like their parent strain.

The growth of four other avirulent strains of tubercle bacilli was inhibited, like that of H37 Ra, by D4, while two slightly virulent strains grew freely in this detergent, like H37Rv. One factor common to the strains naturally insusceptible to D4, but absent from those inhibited, is the ability to produce some degree of microscopic 'cord' pattern, and this property may have some connexion with the cause of the differential inhibition by D4. Surface activity also probably plays a part.

The development of experimental tuberculosis in mice and guinea-pigs has been shown to be markedly suppressed by the injection of non-ionic surfaceactive polyoxyethylene ethers, having the general formula given below, (Cornforth, Hart, Rees \& Stock, 1951; Hart, Long \& Rees, 1952). To obtain these agents, the polymeric condensation products of formaldehyde with

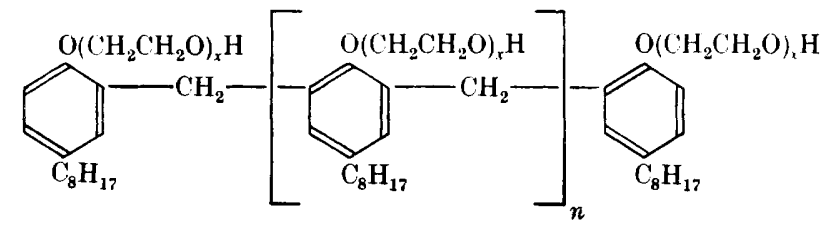

$n=0,1,2,3$, and higher integers. $x=10,20,30$, or 60 (average value).

$p$-tert-octylphenol were made water-soluble by reaction with ethylene oxide, the resulting polyoxyethylene chains being of predetermined average length. For the preparation of the lower members of the series, with one, two or three phenolic nuclei (monocyclics, 'twins' or 'triplets'), the corresponding phenols were available in pure crystalline form, and could be separately condensed with ethylene oxide. The higher members (tetracyclic, etc.) have not so far 
been individually prepared, their precursors being resinous mixtures of polycyclic phenols contaminated with the lower di- and tricyclic phenols.

Of our products in which the polyoxyethylene chains were of medium average length ( $x=20$, see Formula), only the monocyclic compound (D3, see below) appreciably inhibited in vitro the growth of the virulent strain of Mycobacterium tuberculosis $(\mathbf{H} 37 \mathrm{Rv})$ used in previous chemotherapeutic experiments. This compound, however, was not antituberculous in vivo. On the other hand, both the 'twin' (D4) and the 'triplet' (D9a), which were chemotherapeutically inactive, as well as our chemotherapeutically active mixtures of compounds with larger numbers of phenolic nuclei (e.g. D2 and D 2 $a$ ), permitted growth at concentrations of up to $1.0 \%$.

We expected that the detergents would show similar in vitro inertness to avirulent strains of $M$.tuberculosis, but we were aware of the finding of Dubos \& Middlebrook (1948) that at fairly low concentrations the commercial surface-active agent 'Triton A 20' was inhibitory to the avirulent variant, H37 Ra, but not to the virulent variant, H37 Rv. This observation led us to look for a similar selective bacteriostatic property among members of the present series, whose chemical structure is similar to that of 'Triton A 20', but which includes compounds derived from pure phenolic polymers. We were particularly interested in so doing because 'Triton A 20' had toxic properties in the animal not shown by our comparable products, and it seemed possible that this agent's inhibitory power was due to some impurity.

\section{MATERIALS AND METHODS}

Surface-active agents. The chemical structure and average number of ethylene oxide units per phenolic nucleus (see Formula) are given in Table 1, molecular weights to the nearest 10 units being shown in brackets. 'Triton A 20' was made by Rohm and Haas Co., Philadelphia, and supplied by Charles Lennig and Co., Ltd., London; it is stated to be a $25 \%$ solution of an arylalkyl polyether of phenol. All the other products were synthesized by our

Table 1. Details of surface-active agents used*

\begin{tabular}{|c|c|c|c|c|c|c|c|c|}
\hline \multirow[b]{2}{*}{ Chemical structure } & \multicolumn{8}{|c|}{$\begin{array}{l}\text { Average number of ethylene oxide units } \\
\text { per phenolic nucleus }(x)\end{array}$} \\
\hline & \multicolumn{2}{|c|}{10} & \multicolumn{2}{|c|}{20} & \multicolumn{2}{|c|}{30} & \multicolumn{2}{|c|}{60} \\
\hline & \multicolumn{8}{|c|}{ Serial no. (and mean mol. wt.) of detergent } \\
\hline $\begin{array}{l}p \text {-Octylphenol polyoxyethylene } \\
\text { ether (monocyclics) }\end{array}$ & D 15 & $(650)$ & D3 & $(1090)$ & D 19 & $(1530)$ & D 21 & $(2850)$ \\
\hline$n=0$ ('twins') & D 17 & $(1300)$ & D4 & $(2180)$ & D 18 & $(3060)$ & - & - \\
\hline$n=1$ ('triplets') & D 16 & $(1960)$ & D9 $a$ & $(3280)$ & D 13 & $(4600)$ & - & - \\
\hline$n=0,1,2,3$, etc. (mixture) & $\mathrm{D} 14 \dagger$ & - & D2 & - & - & - & D22 & - \\
\hline $\begin{array}{l}\text { As D2 but with less of lower } \\
\text { polymers }\end{array}$ & -- & - & $\mathrm{D} 2 a$ & - & - & - & - & - \\
\hline 'Triton A 20' & - & - & - & - & - & - & - & - \\
\hline
\end{tabular}


colleagues Dr. J. W. Cornforth and Dr J. A. Stock. For bacteriostatic tests, 'Triton A 20' $50 \%(12.5 \%$ effective agent), or our detergents in 6.25 or $12.5 \%(\mathrm{w} / \mathrm{v})$ solutions, were autoclaved, and were used at these strengths or at $1.0 \%$. Such solutions were stable for many months in the refrigerator. The stronger solutions were clear and amber-coloured; those of products with $x=10$ tended to be viscous. Solutions of all the agents at $1 \%$ foamed on shaking. D 14, D2, D2 $a$ and 'Triton A 20' were chemotherapeutically active in experimental tuberculosis in the mouse.

Cultures. The strains of $M$. tuberculosis mainly used were $\mathrm{H37} \mathrm{Rv}$ and $\mathrm{H} 37 \mathrm{Ra}$, virulent and avirulent variants of the human strain $\mathrm{H} \mathrm{37}$; these and four additional avirulent human strains, R1 Ra, JH6 Ra, JH 16 Ra and H4Ra, were obtained from Dr W. Steenken, Jr., Trudeau Laboratory, New York (see Report, 1946; Steenken \& Gardner, 1946a, b; Yegian \& Kurung, 1952). A strain of BCG was obtained from Dr Knud Tolderlund, State Serum Institute, Copenhagen, and the degraded strain VAL from Dr F.C. O. Valentine, The London Hospital. The VAL strain was derived from $\mathrm{H} 37 \mathrm{Rv}$ after repeated subculture in Tween albumin medium; its virulence in mice was considerably less than that of its parent strain, though greater than that of BCG.

Media and tests. The strains of M. tuberculosis were maintained on Herrold's (1931) egg-yolk agar medium, containing $5 \%$ glycerol, or on the surface of Proskauer \& Beck's defined medium containing asparagine (see Baldwin, Petroff \& Gardner, 1927). For bacteriostatic tests, well-grown cultures were ground in Griffith tubes to give aqueous suspensions equal in opacity (by eye) to a standard containing $0 \cdot 2 \mathrm{mg}$. (moist wt.) $/ \mathrm{ml}$. The basal medium used was: Difco Casamino acids, 2.5 g.; asparagine, 0.3 g.; anhydrous $\mathrm{Na}_{2} \mathrm{HPO}_{4}, 2 \cdot 5$ g.; $\mathrm{KH}_{2} \mathrm{PO}_{4}, 1.0$ g.; sodium citrate, 1.5 g.; $\mathrm{MgSO}_{4}, 7 \mathrm{H}_{2} \mathrm{O}, 0.6$ g.; glycerol, $25 \mathrm{ml}$; distilled water to $1000 \mathrm{ml}$. It was distributed in $5 \mathrm{ml}$. quantities in $25 \mathrm{~mm}$. diam. test-tubes and autoclaved, and serial twofold dilutions of the detergents were made, giving concentrations ranging from $2 \cdot 0$ to $0 \cdot 0004 \%(w / v)$. To each tube $0.25 \mathrm{ml} .5 \%$ albumin (bovine plasma fraction $\mathrm{V}$ ) sterilized by filtration, and the inoculum $0.2 \mathrm{ml}$. of a standard suspension $(0.04 \mathrm{mg}$. moist weight of bacilli), were added, and the tubes were incubated at $37^{\circ}$ for 2 weeks. The maximum concentration permitting visible growth, or the minimum inhibitory concentration (which was twice the former value), was taken as end-point (readings made after 3 weeks' incubation did not materially affect the conclusions).

\section{RESULTS}

Effect of the different detergents on the growth of the avirulent strain $\mathrm{H} 37 \mathrm{Ra}$

Bacteriostatic tests were made with the agents having a medium polyoxyethylene chain length $(x=20)$, using the H37 Ra strain and, for comparison, strain H37Rv. Table 2 shows that the monocyclic compound D3 inhibited both strains, the avirulent little more than the virulent. The 'twin' D4, however, provides a striking contrast, this compound markedly inhibiting growth of H37 Ra but not that of H37 Rv. The 'triplet' D9a, on the other hand, was inactive against both avirulent and virulent strains, and so were 
the mixtures D2 and D2a containing higher polymers. The inactivity of D2 indicates a masking effect, since it contains at least $12 \%$ of $\mathrm{D} 4$ as contaminant. The effect of 'Triton A 20 ' is seen to be similar to that of D4, but the contrast is distinctly less. It may be noted that the growth of both strains was submerged in the presence of all the detergents, but that it was granular in D3 and dispersed in the others.

Table 2. Effect of the surface-active agents (medium length of polyoxyethylene chains) on the growth of the virulent strain $H 37 R v$ and the avirulent strain $H 37$ Ra of M. tuberculosis

\begin{tabular}{|c|c|c|c|c|c|c|c|c|}
\hline \multirow{3}{*}{$\begin{array}{c}\text { Final } \\
\text { concentration } \\
\text { in medium } \\
(\%)\end{array}$} & \multicolumn{8}{|c|}{ Surface-active agent } \\
\hline & \multicolumn{2}{|c|}{$\begin{array}{c}\mathrm{D3} \\
\text { (monocyclic) }\end{array}$} & \multicolumn{2}{|c|}{$\frac{\mathrm{D} 4}{\text { ('twin') }}$} & \multicolumn{2}{|c|}{$\begin{array}{l}\text { D9 } a \text { ('triplet'), } \\
\text { D } 2 \text { and D } 2 a \\
\text { (mixtures) }\end{array}$} & \multicolumn{2}{|c|}{ 'Triton A 20'* } \\
\hline & Rv & $\mathbf{R a}$ & Rv & $\mathrm{Ra}$ & Rv & $\mathbf{R a}$ & Rv & $\mathbf{R a}$ \\
\hline 0.5 & 0 & 0 & $++d$ & 0 & $+++d$ & $++d$ & $+d$ & 0 \\
\hline $0 \cdot 2$ & 0 & $\mathbf{0}$ & $++d$ & 0 & $+++d$ & $++d$ & $+d$ & 0 \\
\hline $0 \cdot 1$ & 0 & 0 & $++d$ & 0 & $+++d$ & $++d$ & $++d$ & 0 \\
\hline 0.05 & 0 & 0 & $++d$ & 0 & $+++d$ & $++d$ & $++d$ & 0 \\
\hline 0.025 & 0 & $\mathbf{0}$ & $++d$ & 0 & $+++d$ & $++d$ & $++d$ & 0 \\
\hline $0 \cdot 012$ & $\pm g$ & 0 & $++d$ & 0 & $+++d$ & $++d$ & $+++d$ & tr. d \\
\hline 0.006 & $+\mathrm{g}$ & tr. g & $++d$ & 0 & $+++d$ & $++d$ & $+++d$ & $\pm d$ \\
\hline 0.003 & $++g$ & $\pm \mathrm{g}$ & $t+d$ & 0 & $+++d$ & $++d$ & $+t+d$ & $\overline{+} \mathrm{d}$ \\
\hline $0 \cdot 0015$ & $++\mathrm{g}$ & $+\mathrm{g}$ & $++d$ & tr. gd & $+++g d$ & $t+d$ & $+++d$ & $++d$ \\
\hline $0 \cdot 0008$ & $++\mathrm{g}$ & $+g$ & $++t g$ & $+g$ & $+++g$ & $++g$ & $+++g$ & $++d$ \\
\hline o & $++g$ & $+g$ & $t++g$ & $++g$ & $+++g$ & $t+g$ & $t+t g$ & $++g$ \\
\hline
\end{tabular}

0 , tr.,,,, \pm++++++ indicate degrees of growth; and $\mathrm{g}, \mathrm{d}$, gd, indicate granular, dispersed or partly dispersed type of growth, respectively.

* The concentrations of 'Triton A 20 ' refer to the effective agent.

\section{Effect of varying the length of the polyoxyethylene chains}

The lipophilic character of the molecule of these surface-active agents is determined by the arylalkyl groups. The hydrophilic character depends upon the polyoxyethylene chains, and can be varied by increasing or decreasing the average length of the latter attached by polymerization to a particular crystalline or resinous phenolic material. With this in mind, the phenolic precursors of the medium chain-length products $(x=20), \mathrm{D3}, \mathrm{D} 4, \mathrm{D} 9 a$ and D2, were taken, and ethylene oxide polymerized on to them so that in each case several products were obtained differing only in their (predetermined) lengths of polyoxyethylene chains $(x=10,20,30$ and/or 60$)$ (see Table 1). In this way a range of surface-active agents was created for each of the monocyclic, 'twin' and 'triplet' compounds, and also for the mixture composed mainly of higher polymers. The effect upon the bacteriostatic properties of the members of these four series, when $x$ was increased or decreased from an average of 20 , may be seen in Table 3 and Fig. 1. To show the inhibitory end-points, percentage concentrations (Table 3) have been converted (Fig. 1) into molar concentrations for the monocyclics, 'twins' and 'triplets'. For the higher-polymers 
series an arbitrary molecular weight of $m$ has been assigned to D2, so that the other two variations of the mixture can be related to $\mathrm{D} 2$ also on a molar basis (this is permissible since all three products were derived from the same resin precursor and differ only in the relative amount of polymerized ethylene oxide).

Table 3. Bacteriostatic power shown against strains $H 37 R v$ and $H 37 R a$ by the surface-active agents having varying average lengths of polyoxyethylene chains

Maximum concentration (\%) of detergent permitting growth of tubercle bacilli, virulent (Rv) or avirulent(Ra)

\begin{tabular}{|c|c|c|c|c|c|c|c|c|c|c|c|c|}
\hline \multirow[b]{2}{*}{$x^{*}$} & \multirow{2}{*}{$\begin{array}{l}\text { Mono- } \\
\text { cyclics }\end{array}$} & \multicolumn{2}{|c|}{ Strain } & \multirow[b]{2}{*}{ 'Twins' } & \multicolumn{2}{|c|}{ Strain } & \multirow[b]{2}{*}{ 'Triplets' } & \multicolumn{2}{|c|}{ Strain } & \multirow{2}{*}{$\begin{array}{l}\text { Higher- } \\
\text { polymers } \\
\text { series }\end{array}$} & \multicolumn{2}{|c|}{ Strain } \\
\hline & & Rv & $\mathbf{R a}$ & & $\mathbf{R v}$ & $\mathbf{R a}$ & & $\mathbf{R v}$ & $\mathbf{R a}$ & & Rv & $\mathbf{R a}$ \\
\hline 10 & D15 & $0 \cdot 006$ & 0.003 & D 17 & $0.5+$ & 0.0004 & D 16 & $2 \cdot 0+$ & 0.2 & D14 & $2 \cdot 0+$ & 0.05 \\
\hline 20 & D3 & 0.012 & 0.006 & D4 & $0.5+$ & 0.0015 & $\mathrm{D} 9 a$ & $2 \cdot 0+$ & $2 \cdot 0+$ & D2 & $2 \cdot 0+$ & $2 \cdot 0+$ \\
\hline 30 & D19 & 0.025 & 0.012 & D18 & $0.5+$ & $0.5+$ & D 13 & $2 \cdot 0+$ & $0.5+$ & $\longrightarrow$ & - & - \\
\hline 60 & D21 & $0.5+$ & 0.05 & - & - & - & - & - & - & D 22 & $2 \cdot 0+$ & $\mathbf{2} \cdot \mathbf{0}+$ \\
\hline
\end{tabular}

* Average number ofethylene oxide units/phenolic nucleus. The products listed in any column are derived from the same phenolic precursor and differ only in the value of $x$. (See Formula and Table 1.)

A tendency for inhibitory power (whether measured in percentage or in molar concentrations) to decrease with increasing polyoxyethylene chain length (from $x=10$ to $x=30$ or 60 ) may be seen. For the virulent $\mathrm{H37Rv}$ this is appreciable only in the monocyclic series, where the activity shown by $\mathrm{D} 3(x=20)$ is no longer evident in D21 $(x=60)$, the latter compound failing to inhibit even at the highest concentration tested; on the other hand none of the 'twins', 'triplets' or products of the higher-polymers series were active against this strain over the range of concentrations used. In the case of the avirulent $\mathrm{H37} \mathrm{Ra}$ the association is evident to some degree in each of the series. The inhibitory power of the monocyclic compounds against this strain decreases regularly, though slightly, with increasing chain length (from $x=10$ to $x=60$ ), while in the 'triplets' and also in the higher-polymers mixture the reduction of this length from $x=20$ to $x=10$ is associated with the appearance of inhibition at the highest concentrations used. In the 'twin' series the effect of the association is that the outstanding inhibition of $\mathrm{H3} 7 \mathrm{Ra}$ by $\mathrm{D} 4(x=20)$, already noted, is slightly enhanced when the polyoxyethylene chains are shortened (D 17, $x=10)$, but is lost when they are lengthened to $x=30$ (D 18).

The macroscopic appearances of the submerged growth of both strains, whether granular or dispersed (see Table 2), were unaffected by these alterations of chain length, with two exceptions: (1) partly dispersed growth was given by the Rv strain in the higher concentrations of $\mathrm{D} 21$ and $\mathrm{D} 22$ (the only products with $x=60)$; and (2) the dispersion of the growth given by this strain in D14 $(x=10)$ was unusually fine. Microscopically, the Rv strain produced amorphous clumps when grown in the higher concentrations of the short-chained products D 17, D16 and D14, instead of the well-marked 'cord' formation produced in all the other detergents. The Ra strain grew amorphously in all products. 


\section{Surface activity as a factor in the inhibitory properties of the detergents}

The surface tension of the members of each of the series shown in Fig. 1, measured at fixed molar concentrations selected in the range of the bacteriostatic tests, was found to decrease as polyoxyethylene chain length $(x)$ increased (Cornforth, Hart, Rees \& Stock, in preparation). The fact, already
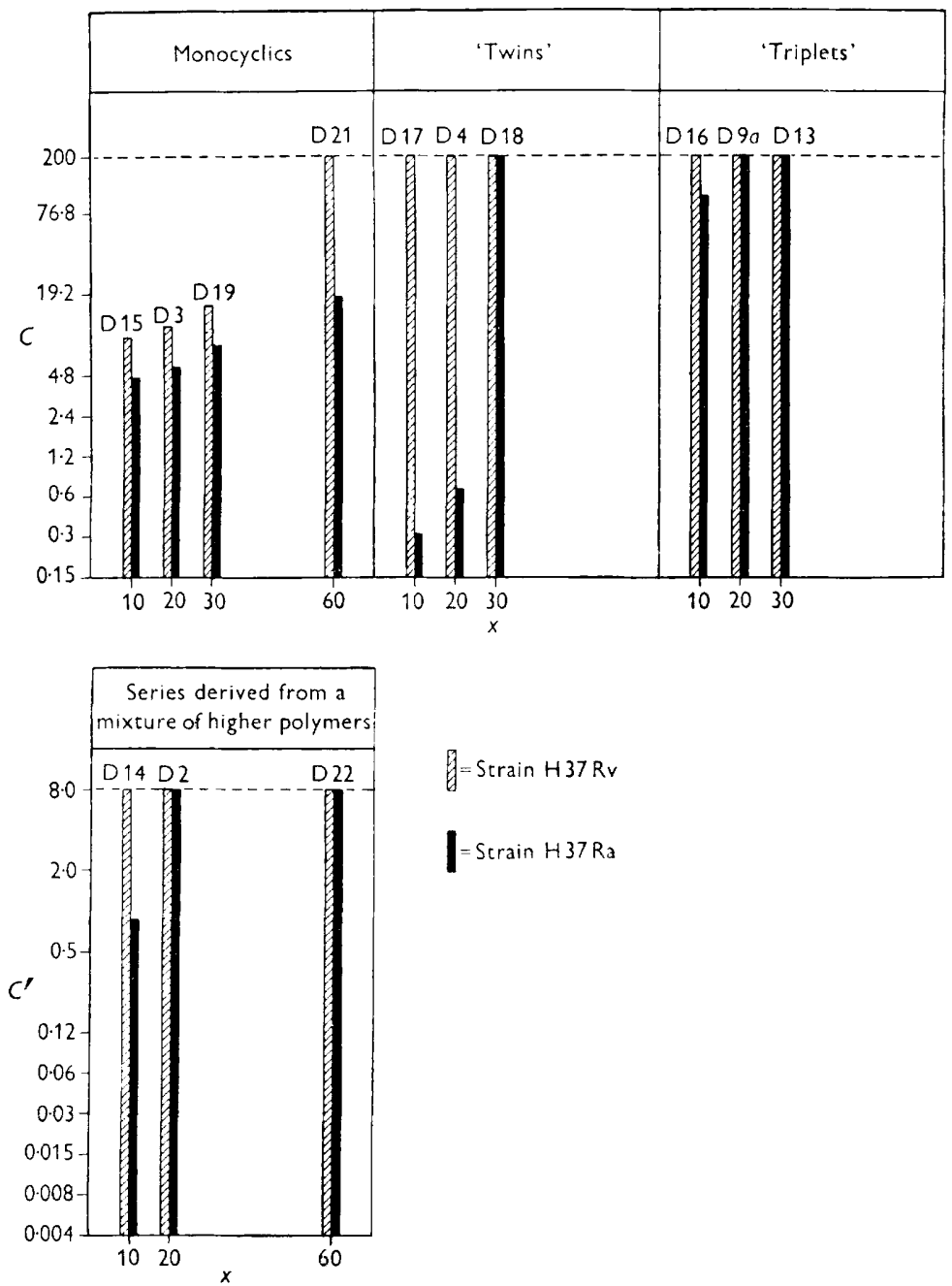

Fig. 1. Bacteriostatic power shown against Mycobacterium tuberculosis, strains $\mathbf{H} 37 \mathrm{Rv}$ and H37 Ra, by the surface-active agents having varying average lengths of polyoxyethylene chains. $C=$ maximum molar concentration $\times 10^{5}$ of detergent permitting growth of tubercle bacilli. $C^{\prime}=$ maximum molar concentration $\times m$ (where $m=$ mean mol. wt. of D2) of detergent permitting growth of tubercle bacilli. $x=$ average number of ethylene oxide units per phenolic nucleus. Note: the horizontal interrupted lines at $200 \times 10^{-5} \mathrm{M}$ and at $\frac{8 \cdot 0}{m} M$ indicate the highest concentrations charted; where $C$ or $C^{\prime}$ is shown at one of these maxima, the actual value was, or may have been, even higher. 
pointed out, that bacteriostatic power against the avirulent strain $\mathrm{H37} \mathrm{Ra}$ also decreased as $x$ increased suggests that the differences in this inhibitory power shown by the various products within each nuclear series (monocyclics, 'twins', etc.) is partly at least determined by differences in their surface activity. However, it is unlikely that surface activity is the main cause of differences between the bacteriostatic power of one nuclear series and that of another, particularly in the strikingly greater inhibition of the growth of H37 Ra by 'twins' than by 'triplets'. Thus, at $25 \times 10^{-5} \mathrm{M}(c .0 \cdot 05 \%)$ the surface tension of the medium-chained 'twin' $\mathrm{D} 4(x=20)$ was $36 \mathrm{dynes} / \mathrm{cm}$., while that of the short-chained 'triplet' D16 $(x=10)$ was 34 dynes $/ \mathrm{cm}$. Yet in spite of its higher surface tension, D4 inhibited the growth of H37 Ra at $25 \times 10^{-5} \mathrm{M}$ and down to $0.69 \times 10^{-5} \mathrm{M}(0.0015 \%)$, whereas D16 permitted growth at $25 \times 10^{-5} \mathrm{M}$ and up to $102 \times 10^{-5} \mathrm{M}(0 \cdot 20 \%)$. A defect of these interpretations is, however, that surface tension indicates only surface activity at the air-liquid interface, and gives no direct information on surface forces at the cell-liquid interface.

\section{Effect of the active 'twin' compounds on other attenuated and avirulent strains of M. tuberculosis}

Each of the four avirulent strains R1 Ra, JH6Ra, JH16 Ra, and H4Ra was inhibited by the compound $\mathrm{D} 4$, much as was $\mathrm{H} 37 \mathrm{Ra}$. In a comparative assay the minimum inhibitory concentrations were, respectively, 0.0015 , $0.006,0.006$ and $0.01 \%$, as compared with $0.003 \%$. As controls, the growth of $\mathrm{H} 37 \mathrm{Rv}$ (inoculum 0.004 as well as the usual $0.04 \mathrm{mg}$.) was, as previously noted, not inhibited by $\mathrm{D} 4$ at $0.2 \%$ (the highest concentration used), and none of the avirulent strains was inhibited by this concentration of D9a or of D 2 $a$. Neither BCG nor the VAL attenuated strain was inhibited to any marked extent by $\mathrm{D} 4$ or by $\mathrm{D} 17$, in the former case even when the inoculum was reduced to $0.004 \mathrm{mg}$. bacilli. Similar negative results were obtained with the saprophytic acid-fast strain ATCC607. The microscopic growth-pattern of these additional avirulent strains in the detergents was amorphous, like that of H37 Ra. On the other hand, the BCG and VAL strains manifested some degree of cord formation, mixed with amorphous or semi-amorphous masses.

\section{Production from $\mathrm{H} 37 \mathrm{R}$ a of strains resistant to certain of the detergents}

Attempts to produce strains from $\mathrm{H37} \mathrm{Rv}$ and $\mathrm{H} 37 \mathrm{Ra}$ able to grow in concentrations of the monocyclic compound D3 greater than the latter's original minimum inhibitory values, using the conventional method of subculturing in increasing drug concentrations, were unsuccessful. However, strains capable of growing in high concentrations of the 'twin' D4 could be rapidly produced in this way from $\mathrm{H} 37 \mathrm{Ra}$. Thus, starting from the tube with the highest concentration of D4 $(0.0015 \%)$ permitting visible growth of $\mathrm{H37} \mathrm{Ra}$ in an initial bacteriostatic assay, there emerged, after 7 serial transfers at 2-4 weeks intervals in increasing concentrations, a strain growing in as much as $2.0 \%$ of this detergent. For an accurate final assay, suspensions prepared from egg-yolk agar cultures of this resistant strain and of the 
unmodified H3r Ra were tested simultaneously for growth inhibition by $\mathrm{D} 4$, using comparable inocula: the inhibitory end-points were found to be $>0 \cdot 2$ and $0.0015 \%$, respectively. In a similar manner, a strain was developed from H37 Ra that would grow in high concentrations of 'Triton A 20'. This strain was found to be also fully resistant to D4, but not to D3. The property of resistance to $\mathrm{D} 4$ and 'Triton A 20 ' of these strains was stable after repeated subculture on egg-yolk agar.

Growth was well dispersed when the D4-resistant and the 'Triton A20' resistant cultures were grown in these two detergents, as was the case with the original sensitive H37 Ra strain. Similarly, also, microscopic examination showed amorphous pattern of the bacilli; however, there was sometimes a tendency to parallelism of the organisms but no definite cord formation. Infection of mice with one of the strains resistant to 'Triton A 20' showed no evidence of enhanced virulence.

\section{DISCUSSION}

We have confirmed the observation of Dubos \& Middlebrook (1948) that the growth of the avirulent strain of tubercle bacillus H $37 \mathrm{Ra}$, but not that of the virulent $H 37 R_{v}$ strain, is inhibited by the non-ionic surface-active agent 'Triton A 20'. Extending this observation by means of the present agents, which form a series differing in the number of phenolic nuclei condensed and subdivided also according to the length of the polyoxyethylene chains, we have found the differential inhibition prominent only in the 'twin' series, but not in either of its neighbours, the monocyclic or the 'triplet' series. Monocyclics were inhibitory to the growth both of the virulent and of the avirulent strains, and this may be accounted for by the generally toxic character of the monocyclic series to biological systems (e.g. to other microorganisms and to experimental animals). On the other hand, the 'triplets" and the mixtures composed mainly of higher polymers showed little or no activity against either strain. Among the active 'twins' we have paid particular attention to the compound $\mathrm{D} 4$, the polyoxyethylene ether of $2,2^{\prime}$-dihydroxy$5,5^{\prime}$-di-tert-octyldiphenylmethane, with an average of twenty polymerized ethylene oxide units per phenolic nucleus.

The connexion between our findings and the inhibitory action of 'Triton A 20' on avirulent tubercle bacilli is not clear. The commercial agent's activity may be due to dicyclic compound, which it contains in significant amount, and in support of this is the cross-resistance to D4 of a 'Triton A 20' resistant strain derived from H37Ra. However, our similar mixtures of higher polymers, such as D2, also contain 'twin' in about the same amount, but the latter's activity is masked therein. This apparent contradiction could be accounted for by other variables, such as differences in the length of polyoxyethylene chains of the components of 'Triton A 20' and D2, respectively, or in preponderance of the various higher polymers.

The main effects of alterations in polyoxyethylene chain length on bacteriostatic activity against $\mathrm{H} 37 \mathrm{Ra}$ are best considered with reference to the product in each nuclear series whose average number of ethylene oxide units 
per phenolic nucleus $(x)$ is 20 . When this medium chain-length compound inhibits H37Ra (as in the 'twin' and monocyclic series), this inhibition is increased when $x$ is decreased below, and is diminished or lost when $x$ is increased above, 20; of particular interest is the absence of activity of the ' $t$ win' compound D 18 which simply differs from the active D4 of the same series by having longer chains. When the medium chain-length compound of a series is inactive against $\mathbf{H 3 7} \mathbf{R a}$ (as in the 'triplet' and higher polymers series), reduction of $x$ to 10 is associated with the appearance of slight inhibitory power. Since changes in $x$ are accompanied by changes in surface tension, these relationships suggest that surface activity, besides influencing dispersion of growth of all strains, contributes to the more specific phenomenon of inhibitory power against avirulent organisms. However, the fact that certain members of the 'twin' series are outstanding in their selective inhibition, but not in their surface tensions, indicates that surface activity is not the only factor responsible.

The bacteriostatic activity of these surface-active agents against avirulent organisms would not be expected to have any relationship to their antituberculous properties. Indeed, both the bacteriostatic D4 and the inert 'triplet' D9a are ineffective in experimental tuberculosis; and while the bacteriostatic 'Triton A20' is antituberculous, so also is the inert mixture D2. There is thus complete lack of correlation.

Although the 'twin' compound $\mathrm{D} 4$, differentiates avirulent tubercle bacilli (H37 Ra and four other well recognized strains) from virulent bacilli ( $\mathrm{H37} \mathrm{Rv}$ ) by the inhibition of the former strains, it does not distinguish between fully virulent and slightly virulent strains, for neither a culture of BCG nor our degraded strain of $\mathrm{H37} \mathrm{Rv}$ (VAL) was inhibited. Indeed, on the basis of present experience, the growth of any strain of tubercle bacillus that shows some degree of 'cord' formation would seem to be unaffected by D4. Yet it is not just this microscopic growth pattern that decides the issue, one way or the other, because avirulent bacilli resistant to 'Triton A20' or to D4, obtained by exposure of $\mathrm{H37} \mathrm{Ra}$ to increasing concentrations of detergent, still grew in a typical amorphous pattern.

Nevertheless, since the only strains of tubercle bacilli so far found to be inhibited by D4 have failed to manifest the slightest cord formation, we suggest as a possibility that the chemical constituents responsible for this growth pattern, and perhaps for virulence as well (see Bloch, 1950; Asselineau \& Lederer, 1951) also protect organisms of lesser or greater virulence from the toxic action of D4. On this hypothesis: (1) the susceptibility of avirulent tubercle bacilli to $\mathrm{D} 4$ is attributable to the ability of this detergent to penetrate readily the cell membrane and then block or interfere with some metabolic process; (2) the production of resistant, but still avirulent, bacilli would be due to the use of some alternative metabolic pathway and (as we have shown) not by emergence of the ability to produce cords; (3) the native resistance of virulent tubercle bacilli to $\mathrm{D} 4$ is attributable to an imperviousness of their surface chemical structure (reflected in their growth pattern) to this detergent. This hypothesis is being tested by further experiments. 
We are indebted to Dr J. W. Cornforth and Dr J. A. Stock for the series of surface-active agents, and to Dr W. Steenken Jr., Dr Knud Tolderlund and Dr F. C. O. Valentine for strains of M. tuberculosis.

\section{REFERENCES}

Asselineau, J. \& Lederer, E. (1951). Recent researches on the chemistry of the lipids of the tubercle bacillus. Experientia, 7, 281.

Baldwin, E. R., Petroff, S. A. \& Gardner, L. U. (1927). Tuberculosis : Bacteriology, Pathology and Laboratory Diagnosis, p. 45. London: Baillière, Tindall and Cox.

BLoci, H. (1950). Studies on the virulence of tubercle bacilli. J. exp. Med. 91, 197.

Cornforth, J. W., Hart, P. D'A., Rees, R. J. W. \& Stock, J. A. (1951). Antituberculous effect of certain surface-active polyoxyethylene ethers in mice. Nature, Lond. 168, 150.

Dubos, R. J. \& Midnlebrook, G. (1948). The effect of wetting agents on the growth of tubercle bacilli. J. exp. Med. 88, 81 .

Hart, P. D'A., Long, D. A. \& Rees, R. J. W. (1952). Depression of tuberculin sensitivity in guinea-pigs by certain antituberculous surface-active agents. Brit. med. J. 1, 680.

Herrold, R. D. (1931). Egg yolk agar medium for the growth of tubercle bacilli. J. infect. Dis. 48, 236.

Repont (1946). A depot for standard cultures of tubercle bacilli. Amer. Rev. Tuberc. $53,511$.

Steenken Jr., W. \& Gardner, L. U. (1946a). R1 strain of tubercle bacillus. Amer. Rev. Tuberc. 54, 51.

Steenken Jr., W. \& Gardner, L. U. (1946b). History of H37 strain of tubercle bacillus. Amer. Rev. Tuberc. 54, 62.

Yegian, D. \& Kurung, J. (1952). Growth-pattern and virulence of tubercle bacilli. Amer. Rev. Tuberc. 65, 181.

(Received 19 May 1952) 